\title{
Property-Optimized Gaussian Basis Sets for Lanthanides
}

Dmitrij Rappoport ${ }^{\mathrm{a})}$

Department of Chemistry, University of California, Irvine, CA 92697, $U S A$

(Dated: 3 August 2021)

Property-optimized Gaussian basis sets of split-valence, triple-zeta and quadruplezeta valence quality are developed for the lanthanides $\mathrm{Ce}-\mathrm{Lu}$ for use with small-core relativistic effective core potentials. They are constructed in a systematic fashion by augmenting def2 orbital basis sets with diffuse basis functions and minimizing negative static isotropic polarizabilities of lanthanide atoms with respect to basis set exponents within the unrestricted Hartree-Fock method. The basis set quality is assessed using a test set of 70 molecules containing the lanthanides in their common oxidation states and $\mathrm{f}$ electron occupations. $5 \mathrm{~d}$ orbital occupation turns out to be the determining factor for the basis set convergence of polarizabilities in lanthanide atoms and the molecular test set. Therefore, two series of property-optimized basis sets are defined. The augmented def2-SVPD, def2-TZVPPD, and def2-QZVPPD basis sets balance the accuracy of polarizabilities across lanthanide oxidation states. The relative errors in atomic and molecular polarizability calculations are $\leq 8 \%$ for augmented split-valence basis sets, $\leq 2.5 \%$ for augmented triple-zeta valence basis sets, and $\leq 1 \%$ for augmented quadruple-zeta valence basis sets. In addition, extended def2-TZVPPDD and def2-QZVPPDD are provided for accurate calculations of lanthanide atoms and neutral clusters. The property-optimized basis sets developed in this work are shown to accurately reproduce electronic absorption spectra of a series of $\mathrm{LnCp}_{3}^{\prime-}$ complexes $\left(\mathrm{Cp}^{\prime}=\mathrm{C}_{5} \mathrm{H}_{4} \mathrm{SiMe}_{3}\right.$, $\mathrm{Ln}=\mathrm{Ce}-\mathrm{Nd}$, Sm $)$ with timedependent density functional theory.

\footnotetext{
a)Electronic mail:dmitrij@rappoport.org
} 


\section{INTRODUCTION}

Lanthanides show a wealth of optical, magnetic, and chemical behaviors due to the presence of partially filled $5 \mathrm{~d}$ and $4 \mathrm{f}$ subshells. The electronic ground states of lanthanide atoms span the range from closed-shell singlet $\left(\mathrm{Yb}^{1} S\right)$ to nonet $\left(\mathrm{Gd}^{9} D^{\circ}\right) \cdot{ }^{1,2}$ The many low-lying excited states, multireference character, and relativistic effects add further complexity to their electronic structures. ${ }^{3-5}$ Both relativistic all-electron methods and effective core potential (ECP) methods are popular in quantum chemical modeling of lanthanide-containing compounds, each having their own atomic basis set requirements. The development of Gaussian basis sets for lanthanides and actinides has been recently reviewed. ${ }^{6-8}$ All-electron Gaussian basis sets for lanthanides include third-order Douglas-Kroll (DK3) basis sets of Hirao and co-workers ${ }^{9,10}$ ANO-RCC basis sets, ${ }^{11}$ SARC basis sets, ${ }^{12,13}$ the segmented-contracted DKH basis set by Dolg, ${ }^{14}$ Sapporo-DK-nZP, ${ }^{15}$ cc-pVnZ basis sets, ${ }^{6,16}$ and DZP and TZP basis sets by Jorge and co-workers. ${ }^{17-19}$ The relativistic basis set family of Dyall and co-workers was developed for four-component Dirac-Hartree-Fock calculations. ${ }^{20}$ More recently, the ANO$\mathrm{R}$ basis sets ${ }^{21}$ and Karlsruhe $\mathrm{x} 2 \mathrm{c}$ basis sets ${ }^{22,23}$ were optimized for the exact two-component (x2c) method. Among ECP approaches, Gaussian basis sets are available for large-core ECPs, which include the $4 \mathrm{f}$ subshell in the atomic core,${ }^{24-27}$ and small-core ECPs, which allow for varying 4 f configurations. ${ }^{28-30}$ The def2 series of segmented contracted basis sets ${ }^{31}$ has been extended to the elements Ce-Lu by Weigend and co-workers ${ }^{7,30}$ for use with small-core ECPs of the Wood-Boring type. ${ }^{32}$

With orbital basis sets designed to accurately reproduce ground-state wavefunctions, the dominant source of errors in calculations of response properties with these basis sets is the lack of low-exponent (diffuse) basis functions. ${ }^{33-35}$ Diffuse basis functions have little influence on the ground-state energies but are crucial for representing the orbital response to external perturbations. At present, diffuse augmentation for lanthanides is rare in the literature. Diffuse basis functions were reported for selected lanthanides by Buchachenko and co-workers. ${ }^{36}$ Sekiya and co-workers obtained diffuse augmentation for their basis sets by downward extrapolation. ${ }^{15}$ Jorge and co-workers developed augmented basis sets from energy

optimizations of atomic anions. ${ }^{18,19}$ The latter two approaches tend to produce extensive augmentation and small diffuse basis set exponents, which impact the efficiency of integraldirect methods and can cause numerical stability problems. 
The construction of property-optimized basis sets derives from the variational property of static polarizabilities. ${ }^{37}$ Diffuse augmentation of property-optimized basis sets is obtained by minimizing a target quantity, namely the negative static Hartree-Fock (HF) polarizability, with respect to basis set parameters. The basis set optimization procedure is equivalent to that for energy-optimized orbital basis sets, in which the ground-state HF energy is typically the target quantity. ${ }^{30,31,33-35}$ As we previously reported, ${ }^{37}$ the bulk of the basis set errors in static polarizabilities is addressed by adding only few diffuse basis functions with optimized exponents. The differential contributions of a second, third, etc., sets of basis functions with the same angular momentum $(l)$ quantum number and optimized exponents decrease exponentially, making multiple augmentation usually unnecessary. We also found that the diffuse augmentation in these basis sets does not need to increase with the size of the underlying orbital basis set.

This paper reports property-optimized augmented Gaussian basis sets of split-valence, triple-zeta and quadruple-zeta valence quality for the elements Ce-Lu. The diffuse augmentation is obtained by minimizing negative static HF polarizabilities of the Ce-Lu atoms in their $4 \mathrm{f}^{n} 5 \mathrm{~d}^{1}(n=1-14)$ and $4 \mathrm{f}^{n+1}(n=2-13)$ configurations. We assess the accuracy of the property-optimized basis sets using an extended version of the molecular test set of Weigend and co-workers, which includes common oxidation states of the lanthanides. ${ }^{7,30}$ Our construction of property-optimized basis sets aims for $\leq 8 \%$ target accuracy for augmented split-valence basis sets, $\leq 2.5 \%$ for augmented triple-zeta valence basis sets, slightly relaxed from the target of $\leq 2 \%$ used in our previous work, and $\leq 1 \%$ for augmented quadruple-zeta valence basis sets. However, the selection of diffuse augmentations for the lanthanides is complicated by the strong and somewhat unexpected sensitivity of the basis set requirements in lanthanides to their oxidation states and $5 \mathrm{~d}$ occupations. In order to balance accuracy and basis set size, we define two series of property-optimized augmented basis sets for Ce-Lu. The def2-SVPD, def2-TZVPPD, and def2-QZVPPD basis sets provide economic augmentation and balance the accuracy of polarizabilities across lanthanide oxidation states. In addition, the def2-TZVPPDD and def2-QZVPPDD include larger diffuse sets and are only needed for accurate calculations of lanthanide atoms and neutral clusters.

This paper is structured as follows. We briefly describe the optimization procedure for property-optimized basis sets in Section II. The resulting basis sets and their basis set convergence are discussed in Section III. The use of property-optimized basis sets is illus- 
trated in Section IV for electronic absorption spectra of a series of $\mathrm{LnCp}_{3}^{\prime-}$ complexes $\left(\mathrm{Cp}^{\prime}\right.$ $\left.=\mathrm{C}_{5} \mathrm{H}_{4} \mathrm{SiMe}_{3}, \mathrm{Ln}=\mathrm{Ce}-\mathrm{Nd} \mathrm{Sm}\right) \cdot{ }^{38-40}$ We close with a discussion in Section $\mathrm{V}$ and present our conclusions in Section VI.

\section{METHODS}

The construction of property-optimized basis sets is described in Ref. 37, to which we refer for details. Only a brief discussion will be given here. The basis set optimization procedure relies on the variational property of static polarizabilities. Specifically, the negative $m n$ component of the static polarizability, $-\alpha^{m n}(m, n=x, y, z)$, is the stationary point of the Hylleraas functional ${ }^{41,42}$

$$
G\left[\tilde{\Psi}^{m}, \tilde{\Psi}^{n}\right]=\left\langle\tilde{\Psi}^{m}\left|\left(H^{(0)}-E^{(0)}\right)\right| \tilde{\Psi}^{n}\right\rangle+\left\langle\tilde{\Psi}^{m}\left|H^{n}\right| \Psi^{(0)}\right\rangle+\left\langle\Psi^{(0)}\left|H^{m}\right| \tilde{\Psi}^{n}\right\rangle
$$

where $\left|\Psi^{(0)}\right\rangle$ is an eigenfunction of the unperturbed Hamiltonian $H^{(0)}$ with the corresponding energy eigenvalue $E^{(0)}$, and the operators $H^{m}, H^{n}$ are the $m$ and $n$ components of the electric dipole perturbation. If the functional $G\left[\tilde{\Psi}^{m}, \tilde{\Psi}^{n}\right]$ is a positive definite bilinear form, which is the case when $\left|\Psi^{(0)}\right\rangle$ is the ground state of the Hamiltonian $H^{(0)}$ (or the lowest-energy state within a given symmetry representation), then the stationary point is a minimum,

$$
G\left[\tilde{\Psi}^{m}, \tilde{\Psi}^{n}\right] \geq G\left[\Psi^{m}, \Psi^{n}\right]=-\alpha^{m n}
$$

where $\left|\Psi^{m}\right\rangle$ and $\left|\Psi^{n}\right\rangle$ correspond to the first-order wavefunction response to the dipole perturbations $H^{m}$ and $H^{n}$, respectively. This variational condition allows us to obtain property-optimized basis sets by minimizing negative static polarizabilities with respect to basis set parameters. As in our previous work ${ }^{37}$ the basis set optimization procedure utilizes the static isotropic polarizabilities $\alpha_{\text {iso }}=\frac{1}{3} \sum_{m=x, y, z} \alpha^{m m}$ of lanthanide atoms within the unrestricted HF (UHF) method.

We note that the Hylleraas functional is not variational with respect to the unperturbed electronic state. The strict application of the minimum property of Eq. 2 thus requires that the basis set is held constant in ground-state UHF calculations and that dual-basis techniques are used for computing polarizabilities. ${ }^{43,44}$ Indeed, excessive diffuse augmentation leads to an admixture of excited electronic states to the basis set representation of the ground-state wavefunction, which has a negligible effect on energies but presents itself as overpolariza- 
tion. ${ }^{45}$ However, we find that this issue rarely presents problems in property-optimized basis sets with moderate diffuse augmentation.

The reference UHF states of the atoms Ce-Lu with def2-SVP basis $\operatorname{sets}^{30}$ and smallcore ECPs $^{32}$ were determined by exhaustive search over atomic orbital (AO) occupations in $D_{2 h}$ symmetry. AO occupations corresponding to the $4 \mathrm{f}^{n} 5 \mathrm{~d}^{1}$ configuration were obtained for $\mathrm{Ce}-\mathrm{Sm}, \mathrm{Gd}-\mathrm{Tm}, \mathrm{Lu}(n=1-6,8-12,14)$. Reference states corresponding to the $4 \mathrm{f}^{n+1}$ configuration were determined for $\mathrm{Pr}-\mathrm{Eu}, \mathrm{Tb}-\mathrm{Yb}(n=2-6,8-13)$. The AO occupations and UHF energies of the reference states are given in the Section S1 of the Supplementary Material (SM).

In the construction of the property-optimized basis sets for the lanthanides, the def2-SVP, def2-TZVPP, and def2-QZVPP orbital basis sets ${ }^{30}$ were successively augmented with uncontracted diffuse basis functions with $l=0-4(\mathrm{~s}, \mathrm{p}, \mathrm{d}, \mathrm{f}, \mathrm{g})$ angular momentum quantum numbers. The static UHF polarizabilities were evaluated using nonorthonormal Krylovspace methods ${ }^{46}$ with $10^{-10}$ convergence threshold for the residual norm. The exponents of the diffuse basis functions were optimized for each augmentation pattern by minimizing the negative logarithmic UHF polarizabilities $\left(-\log \alpha_{\text {iso }}\right)$ of the atoms. The convergence criteria for the basis set optimization were $10^{-8}$ a.u. for $\left(-\log \alpha_{\text {iso }}\right)$ and $10^{-5}$ a.u. for its gradient. The polarizability derivatives with respect to basis set parameters were evaluated numerically using a 4-point central difference formula with $10^{-4}$ a.u. spacing. In the following we use a compact notation to describe diffuse augmentation. A 1p diffuse set, for example, denotes a complete set of diffuse basis functions with angular momentum quantum number $l=1(\mathrm{p})$. The basis set limit in atomic calculations was approximated by def2-QZVPP basis including $2 \mathrm{~s} 2 \mathrm{p} 2 \mathrm{~d} 2 \mathrm{f} 2 \mathrm{~g}$ augmentation from even-tempered downward extrapolation.

Since the minimum property of the Hylleraas functional $G\left[\tilde{\Psi}^{m}, \tilde{\Psi}^{n}\right]$ relies on the reference state $\left|\Psi^{(0)}\right\rangle$ being stable, ${ }^{47-49}$ the optimization procedure fails in the presence of reference state instabilities. We observed electronic instabilities with UHF for the Tm $4 \mathrm{f}^{12} 5 \mathrm{~d}^{1}$ and Lu $4 \mathrm{f}^{14} 5 \mathrm{~d}^{1}$ reference states, which could not be remedied by symmetry breaking. The basis set optimization procedure fails in these cases. In the $\operatorname{Tm} 4 \mathrm{f}^{12} 5 \mathrm{~d}^{1}$ reference state, the basis set exponents were determined by extrapolation from Ce-Sm, Gd-Er $4 \mathrm{f}^{n} 5 \mathrm{~d}^{1}(n=1-11)$ states, while basis set optimizations for the $\mathrm{Lu} 4 \mathrm{f}^{14} 5 \mathrm{~d}^{1}$ succeeded with $1 \mathrm{f}$ diffuse augmentation. See Section III for details.

The molecular test set of Weigend and co-workers ${ }^{30}$ was extended in this work to 70 
molecules covering the elements $\mathrm{Ce}-\mathrm{Lu}$ in their common oxidation states and $\mathrm{f}$ electron occupations. The dioxides $\mathrm{CeO}_{2}$ and $\mathrm{TbO}_{2}$ were included as representatives of the +4 oxidation state. The set of lanthanide dimers was extended by the early-lanthanide molecules $\mathrm{Ce}_{2}$ and $\mathrm{Pr}_{2}$. Several additions were made to obtain complete coverage of the low-valent states of Tb, Ho, Er as well as Pm, Eu, Tb, Tm, Yb +3 oxidation states. For consistency with Ref. 30, the structures of the added molecules were optimized by density functional theory (DFT) with the BP86 exchange-correlation functional, ${ }^{50,51}$ the Cao-Dolg basis sets ${ }^{29}$ for the lanthanides, and def2-QZVP basis $\operatorname{sets}^{52}$ for all other atoms. The structures of some molecules in the test set were symmetrized and re-optimized based on the available literature data. The orbital occupations, spin expectation values, and UHF energies of the extended molecular test set are given in the Section S3 of the SM. 5d and 4f orbital occupations from natural population analysis ${ }^{53}$ are also included. The Cartesian coordinates of the optimized molecular structures are provided in the Section S5 of the SM. Calculations with def2-QZVPP basis sets and 1s1p1d1f1g extrapolated augmentations for all elements (1s1p1d1f for H) were used to approximate the basis set limits of molecular polarizabilities. All calculations were performed with the Turbomole program package, version $7.5 .{ }^{54,55}$

\section{RESULTS}

The competition between the $4 \mathrm{f}$ and $5 \mathrm{~d}$ subshells is a characteristic feature of lanthanides. The experimental ground states of the elements Ce, Gd, and $\mathrm{Lu}$ have $4 \mathrm{f}^{n} 5 \mathrm{~d}^{1}$ configurations $(n=1,7,14)$. The elements $\mathrm{Pr}-\mathrm{Eu}$ and $\mathrm{Tb}-\mathrm{Yb}$ have the ground state configurations $4 \mathrm{f}^{n+1}$ with $n=2-6,8-13$, however, with the exceptions of $\mathrm{Eu}$ and $\mathrm{Yb}$, the corresponding $4 \mathrm{f}^{n} 5 \mathrm{~d}^{1}$ configurations are close in energy. The atomic states arising from $4 \mathrm{f}^{n} 5 \mathrm{~d}^{1}$ and $4 \mathrm{f}^{n+1}$ configurations show drastically different convergence of their static isotropic polarizabilities $\alpha_{\text {iso }}$, as illustrated in Fig. 1 for the Pr atom. The polarizability of the $\operatorname{Pr} 4 \mathrm{f}^{3}$ state is ca. 11\% away from the basis set limit with the unaugmented def2-SVP and def2-TZVPP basis sets and is still $4 \%$ in error with the def2-QZVPP basis set, see Fig. 1(a). However, the basis set limit is reached with the addition of a $1 p$ diffuse set, with all augmented basis sets having less than $1 \%$ relative error. This is line with our previous results for the neighboring elements $\mathrm{Ba}$ and La, which converge quickly to the basis set limit and require at most 1p diffuse augmentation. ${ }^{37}$ 


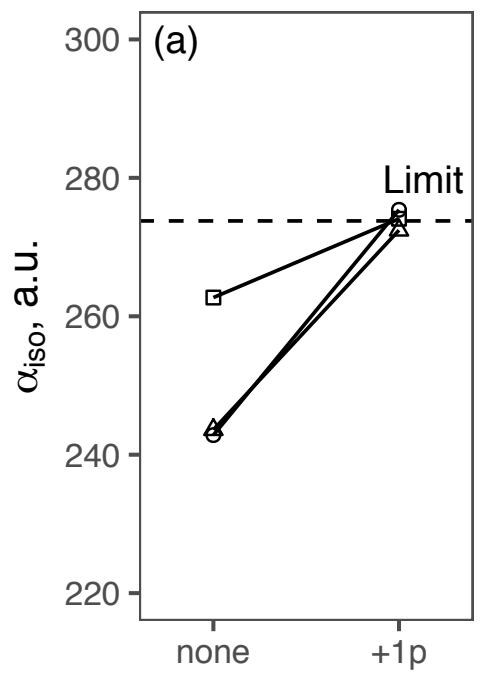

Augmentation

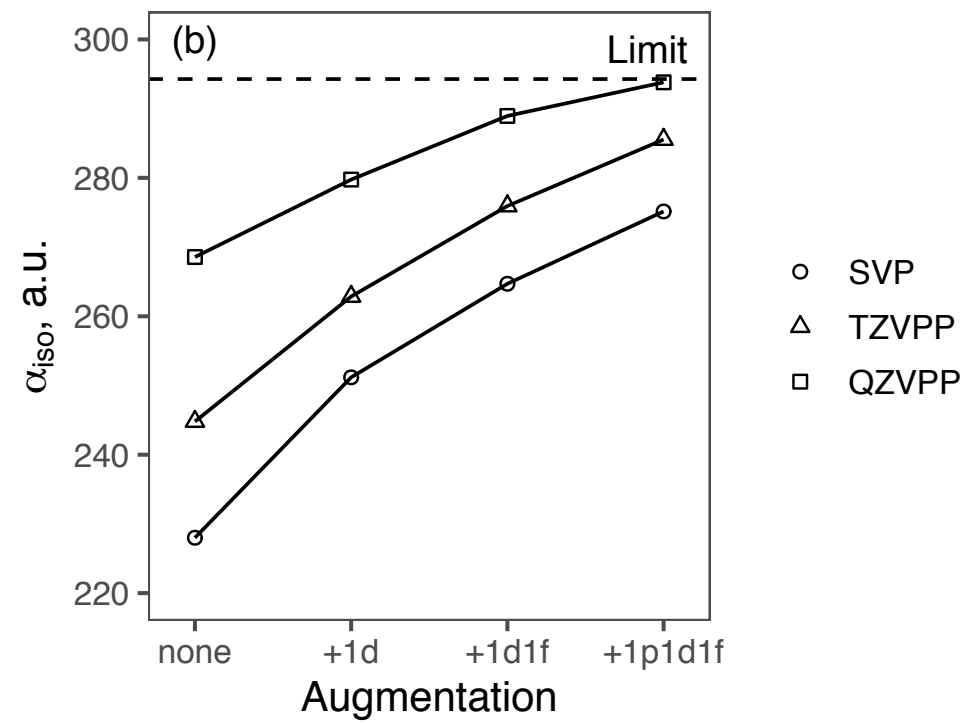

FIG. 1. Basis set convergence of static isotropic HF polarizability $\alpha_{\text {iso }}$ of the Pr atom with diffuse augmentation using optimized exponents in the (a) $4 \mathrm{f}^{3}$ and (b) $4 \mathrm{f}^{2} 5 \mathrm{~d}^{1}$ states. Horizontal dashed lines represent the basis set limits.

By contrast, the Pr $4 \mathrm{f}^{2} 5 \mathrm{~d}^{1}$ state shows very slow convergence to the basis set limit, see Fig. 1(b). The def2-SVP basis set has a $22.5 \%$ relative error compared to the basis set limit, while the def2-QZVPP basis set still $8.7 \%$ away from the limit. The largest contributions to the polarizability of the $\operatorname{Pr} 4 \mathrm{f}^{2} 5 \mathrm{~d}^{1}$ state are, in decreasing order, from 1d, $1 \mathrm{f}$, and $1 \mathrm{p}$ diffuse sets. The 1p1d1f augmented double-zeta, triple-zeta valence, and quadruple-zeta valence basis sets have $6.5 \%, 3.0 \%$, and $0.2 \%$ relative errors, respectively. These trends continue throughout the lanthanide series, as shown in the Section S2 of the SM. The polarizabilities of $4 \mathrm{f}^{n+1}$ states convergence quickly towards the basis set limit, similar to that of Ba and La. The unaugmented basis sets already yield relatively small basis set errors, while the addition of a $1 \mathrm{p}$ diffuse set is sufficient to reach the basis set limit. The $4 \mathrm{f}^{n} 5 \mathrm{~d}^{1}$ states are much more problematic. As a consequence of the $5 \mathrm{~d}$ occupation, diffuse $\mathrm{d}$ and $\mathrm{f}$ functions are required to accurately capture the orbital response in these states. The largest basis set errors are observed in the early lanthanides $\mathrm{Ce}-\mathrm{Nd}$, in which $5 \mathrm{~d}$ orbitals have the largest spatial extent due to lanthanide contraction. ${ }^{6}$

The construction of the def2-SVP basis sets for lanthanides by removing one basis function each from the $\mathrm{p}, \mathrm{d}$, and $\mathrm{f}$ spaces and re-optimizing ${ }^{30}$ produces a gap in p basis set exponents in some lanthanide atoms. For $\mathrm{Ce}-\mathrm{Nd}$ and $\mathrm{Ho}$, the ratios between the smallest and the second- 
smallest $\mathrm{p}$ function exponents in their def2-SVP basis are greater than 5 . The unconstrained optimization of def2-SVP basis sets for lanthanides (except for Pr, Eu, Yb) and def2-TZVPP basis sets of $\mathrm{Nd}, \mathrm{Sm}, \mathrm{Tb}-\mathrm{Er}$ with 1p augmentation thus causes the p basis function exponents to intrude into the valence/polarization space. In these cases, the diffuse p basis function exponents were fixed to $\frac{1}{2} \zeta_{p, \text { min }}$, where $\zeta_{p \text {,min }}$ is the smallest $\mathrm{p}$ function exponent of the orbital basis set. Additionally, we investigated the effect of inserting a 1p polarization set into def2SVP and def2-TZVPP basis sets with exponents obtained by interpolation. The results are included in the Section S1 of the SM. The addition of the 1p polarization set accelerates the convergence of atomic polarizabilities with augmented def2-SVP and def2-TZVPP basis sets but was found to have only a modest effect in the molecular test set. This avenue was not further pursued in this work.

The $\mathrm{Tm} 4 \mathrm{f}^{12} 5 \mathrm{~d}^{1}$ and $\mathrm{Lu} 4 \mathrm{f}^{14} 5 \mathrm{~d}^{1}$ states pose a different set of issues due to electronic instabilities in their UHF wavefunctions. The instability of the Tm $4 \mathrm{f}^{12} 5 \mathrm{~d}^{1}$ state shows up in the very large polarizabilities with augmented basis sets, which far exceed those of the neighboring elements Er and Yb, see Section S2 of the SM. Moreover, the addition of diffuse $\mathrm{f}$ basis functions leads to negative static polarizability components. We note that all electronic states corresponding to the $\mathrm{Tm} 4 \mathrm{f}^{12} 5 \mathrm{~d}^{1}$ configuration were enumerated and shown to suffer from the instabilities described here. The exponents of the diffuse basis functions for Tm were thus obtained by extrapolation from optimized Ce-Sm, Gd-Er basis sets. In $\mathrm{Lu} 4 \mathrm{f}^{14} 5 \mathrm{~d}^{1}$ states, the static polarizability components turn negative with def2-TZVPP and def2-QZVPP basis sets. The addition of $1 \mathrm{f}$ diffuse sets removes the instabilities, while basis functions with other $l$ quantum numbers do not seem to have an appreciable effect on the polarizability. However, the basis set convergence of the polarizability of the $\mathrm{Lu} 4 \mathrm{f}^{14} 5 \mathrm{~d}^{1}$ state is not monotonic and the corresponding basis set error estimates are not reliable.

Given that $4 \mathrm{f}^{n} 5 \mathrm{~d}^{1}$ configurations correspond to excited states in lanthanide atoms (except $\mathrm{Ce}, \mathrm{Gd}$, and $\mathrm{Lu}$ ) and that the occupation of diffuse $5 \mathrm{~d}$ orbitals leads to slow basis set convergence of response properties, it might be tempting to leave these states out from the construction of property-optimized basis sets. $5 \mathrm{~d}$ orbital occupation is not limited to neutral lanthanide atoms, however. On the contrary, 5d orbitals are occupied in lanthanide clusters $^{56-59}$ and contribute to bonding in low-valent small molecules ${ }^{4,60,61}$ and a growing number of organometallic complexes ${ }^{40,62-67}$ of lanthanides. In selecting the augmentation patterns for the property-optimized basis sets, we have to take into consideration the basis 
set convergence in both $4 \mathrm{f}^{n} 5 \mathrm{~d}^{1}$ atomic states (Ce-Sm, Gd-Tm, Lu, $n=1-6,8-12,14$ ) and $4 \mathrm{f}^{n+1}$ atomic states $(\mathrm{Pr}-\mathrm{Eu}, \mathrm{Tb}-\mathrm{Yb}, n=2-6,8-13)$ as well as in the molecular test. The molecular test set results display a large variation across the lanthanide series and as a function of the lanthanide oxidation state. We aim for a target accuracy of $\leq 8 \%$ for augmented split-valence basis sets, $\leq 2.5 \%$ for augmented triple-zeta valence basis sets, and $\leq 1 \%$ for augmented quadruple-zeta valence basis sets. We give a brief summary of the trends in the molecular test set in the following. The complete molecular test results are compiled in the Section S4 of the SM.

a. $C e-\operatorname{Pr}$ The neutral dimers $\mathrm{Ce}_{2}$ and $\mathrm{Pr}_{2}$ are derived from the $4 \mathrm{f}^{n-1} 5 \mathrm{~d}^{2}$ atomic states $(n=1-2)^{56-59,68}$ and show similarly slow basis set convergence to the $4 \mathrm{f}^{n} 5 \mathrm{~d}^{1}$ states of the neutral atoms. 1p1d1f diffuse augmentation is necessary to reach the target accuracy. The example of $\mathrm{Pr}_{2}$ also illustrates the importance of balancing the contributions from diffuse basis functions of different $l$ quantum numbers. def2-SVP basis sets with the Pr $4 \mathrm{f}^{3}$ statebased 1p diffuse augmentation overestimate the basis set limit in $\operatorname{Pr}_{2}$ by $15 \%$, while the $\operatorname{Pr} 4 \mathrm{f}^{2} 5 \mathrm{~d}^{1}$-based 1p1d1f augmentation yields an error of only $1.8 \%$. Low-valent Ce and $\operatorname{Pr}$ compounds with lanthanide atoms in the +1 and +2 oxidation states show faster basis set convergence than the neutral lanthanide dimers due to only partial 5d occupations. 1d1f diffuse augmentation is generally sufficient to achieve target accuracy, while the effect of diffuse $\mathrm{p}$ functions is negligible in divalent compounds. The compounds of $\mathrm{Ce}$ and $\operatorname{Pr}$ in their most common +3 oxidation state are highly ionic and contain $\mathrm{Ln}^{+3}$ cations with $4 \mathrm{f}^{n}$ configurations $(n=1-2) \cdot{ }^{69-72}$ These compounds behave similarly to alkali and earth alkali metal salts, in which the basis set convergence of polarizabilities is dominated by the negatively charged counterions, while diffuse augmentation on the metal atoms has essentially no effect. ${ }^{37}$ Likewise, diffuse augmentation of the Ce atom in $\mathrm{CeO}_{2}$ (oxidation state +4$)$ contributes only little to its polarizability.

b. $\quad \mathrm{Nd}-\mathrm{Sm}$ Compared to $\mathrm{Ce}-\mathrm{Pr}$, the basis set requirements of the following elements $\mathrm{Nd}-\mathrm{Sm}$ are reduced due to their more compact valence orbitals. ${ }^{1,6}$ In particular, diffuse $\mathrm{p}$ and $\mathrm{f}$ basis functions make relatively small contributions to polarizabilities in low-valent compounds of these elements. The diffuse augmentations optimized using $4 \mathrm{f}^{n+1}$ reference states $(n=3-5)$ are thus unsuitable for molecular calculations. 1d augmented basis sets (optimized using $4 \mathrm{f}^{n} 5 \mathrm{~d}^{1}$ reference states, $n=3-5$ ) give on average the desired accuracy. Within this average, however, the effect of diffuse 1d augmentation decreases considerably 
with the lanthanide oxidation state. The compounds of $\mathrm{Nd}-\mathrm{Sm}$ in their +1 and +2 oxidation states reach the target accuracy with 1d diffuse augmentation, while the ionic trivalent compounds require no diffuse augmentation at all on the lanthanide atoms.

c. $E u, Y b$ Due to the presence of half-filled or completely filled $4 \mathrm{f}$ shells, Eu and $\mathrm{Yb}$ have the highest $4 \mathrm{f} \rightarrow 5 \mathrm{~d}$ promotion energies of the lanthanide series. ${ }^{1}$ Because $5 \mathrm{~d}$ orbitals are unoccupied in Eu and $\mathrm{Yb}$ compounds, we observe rapid basis set convergence, in line with that of $\mathrm{Ba}$ and La compounds. ${ }^{37}$ Similar to the earth alkali dimers, the $\mathrm{Eu}_{2}$ and $\mathrm{Yb}_{2}$ molecules are predominantly van der Waals bound. ${ }^{56,73,74}$ The effect of 1 p diffuse augmentation in Eu and $\mathrm{Yb}$ compounds is only noticeable when def2-TZVPP and def2-QZVPP basis sets are used and higher accuracy is desired. The ionic compounds of Eu and $\mathrm{Yb}$ in +2 and +3 oxidation states do not require diffuse augmentation on the lanthanide atoms.

d. $G d-H o$ The basis set convergence in compounds of $\mathrm{Gd}-\mathrm{Ho}$ is similar to that in $\mathrm{Nd}-\mathrm{Sm}$. The neutral dimer $\mathrm{Gd}_{2}$ is characterized by very slow basis set convergence and requires 1p1d1f diffuse augmentation to reach target accuracy. However, the compounds of Gd-Ho in positive oxidation states are well described using 1d diffuse augmentation. The trihalides of $\mathrm{Gd}-\mathrm{Ho}$ require no diffuse augmentation on the lanthanide atoms at all. $\mathrm{TbO}_{2}$ contains $\mathrm{Tb}$ in the oxidation state +4 and shows rapid basis set convergence, apart from the anomalous behavior of augmented def2-SVP basis sets, which significantly overestimate its polarizability.

e. Er-Tm The compounds of the late lanthanides Er-Tm show the fastest basis set convergence of the lanthanide-containing molecules with $5 \mathrm{~d}$ occupations. Like many other trends in the basis set requirements of lanthanides, this effect can be attributed to the lanthanide contraction, which reduces the spatial extent of $5 \mathrm{~d}$ orbitals in these elements. $1 \mathrm{~d}$ diffuse augmentation achieves the target accuracy on average for the molecules containing Er-Tm.

f. $\quad L u$ The properties of $\mathrm{Lu}$ set it apart from the other lanthanides and are subject to a protracted debate about whether it should even be included in the lanthanide series. ${ }^{75}$ With respect to the basis set convergence, the $\mathrm{Lu} 4 \mathrm{f}^{14} 5 \mathrm{~d}^{1}$ state is distinct from the other $4 \mathrm{f}^{n} 5 \mathrm{~d}^{1}$ atomic states of lanthanides and requires $1 \mathrm{f}$ diffuse augmentation. Lu also differs from the neighboring elements $\mathrm{Hf}$ and $\mathrm{Nb}$, which, like other transition metals, depend on $1 \mathrm{p}$ diffuse augmentation. ${ }^{37}$ The instabilities of the Lu $4 \mathrm{f}^{14} 5 \mathrm{~d}^{1}$ state with UHF make the basis set convergence difficult to quantify, as discussed above. However, the polarizabilities of Lu 
compounds are within target accuracy without diffuse augmentation on the Lu atom, with the exception of the $\mathrm{Lu}_{2}$ dimer, which reaches the target accuracy with if augmentation.

The large variation in the augmentation requirements in lanthanide atoms and lanthanidecontaining molecules leads us to deviate from the scheme we developed in our previous work for the main-group elements and transition metals and to define two series of propertyoptimized augmented basis sets for Ce-Lu. The generally recommended def2-SVPD, def2TZVPPD, and def2-QZVPPD basis sets are designed for calculations of compounds containing lanthanides in positive oxidation states. They are constructed to yield the target accuracy on average across the molecular test set. In addition, we define extended def2TZVPPDD and def2-QZVPPDD basis sets, which are suitable for accurate studies of lanthanide atoms and neutral clusters and yield the target accuracy specifically for lanthanide atoms in $4 \mathrm{f}^{n} 5 \mathrm{~d}^{1}$ and $4 \mathrm{f}^{n+1}$ atomic states and in neutral metal dimers. The augmentation patterns of the property-optimized basis sets are shown in Table I. The statistics of relative errors of static isotropic UHF polarizabilities are presented in Table II for the atoms Ce- $\mathrm{Yb}$ in their $4 \mathrm{f}^{n} 5 \mathrm{~d}^{1}$ and $4 \mathrm{f}^{n+1}$ states (except Tm $4 \mathrm{f}^{12} 5 \mathrm{~d}^{1}$ ). The relative errors of static isotropic UHF polarizabilities of the molecular test set are shown in Table III. Fig. 2 summarizes the molecular test results by lanthanide oxidation state. See Section S2 of the SM for the complete data.

The default augmented basis sets def2-SVPD, def2-TZVPPD, and def2-QZVPPD are designed for all lanthanide calculations except in atoms and neutral metal clusters. These basis sets contain 1d1f diffuse augmentation for Ce and Pr and 1d augmentation for the later lanthanides Nd-Sm, Gd-Tm. As in our previous work, ${ }^{37}$ the diffuse set does not increase with the size of the underlying basis set. For Eu and Yb, diffuse augmentation is only added to the def2-QZVPP basis sets in order to obtain the target accuracy of $\leq 1 \%$ basis set error. No augmentation is included in the Lu basis sets. The mean unsigned error (MUE) with the def2-SVPD basis sets is $8.0 \%$ for the atomic polarizabilities and $4.4 \%$ for the molecular test set relative to the basis set limit, compared to $12.2 \%$ MUE for atomic calculations and $22.6 \%$ MUE for the molecular test set using the unaugmented def2-SVP basis sets, see Tables II and III. With the def2-TZVPPD and def2-QZVPPD basis sets, the MUEs for the molecular test set are $1.9 \%$ and $0.9 \%$, respectively.

Fig. 2 offers a more detailed view of the relative errors of static isotropic UHF polarizabilities within the molecular test set by oxidation state using box-and-whiskers plots. The 
median error within each group is shown by a thick horizontal line, while the box covers the range between the first and third quartiles (interquartile range, IQR). The vertical lines (whiskers) show minimum and maximum values, excluding outliers. Outliers are defined as data points lying further than 1.5 times the IQR outside the box and are shown by empty circles. The MUE of monovalent lanthanide compounds with def2-SVPD basis sets is $4.1 \%$, that of divalent lanthanide compounds is $4.4 \%$, and that of tri- and tetravalent lanthanide compounds is $3.2 \%$, similar to each other and well within the target accuracy, see Fig. 2(b)-(d). However, the MUE for def2-SVPD basis sets in neutral lanthanide dimers in Fig. 2(a) is much larger, with $10.0 \%$ on average and the largest deviation of $18.8 \%$ found in $\mathrm{Gd}_{2}$. Similarly, for def2-TZVPPD basis sets the overall MUE is 1.9\%. Within this average statistic, basis set convergence is essentially reached for the tri- and tetravalent lanthanide compounds, which are only $0.4 \%$ away from the basis set limit. The MUEs for monovalent and divalent lanthanide compounds with def2-TZVPPD basis sets are $1.4 \%$ and $1.9 \%$, respectively. At the same time, the zero-valent lanthanide compounds still have an unacceptably high 8.4\% MUE with these basis sets. The overall MUE for def2-QZVPPD basis sets is $0.9 \%$. This average encompasses the 3.3\% MUE for zero-valent, $1.0 \%$ MUE for monovalent, $0.9 \% \mathrm{MUE}$ for divalent, and a negligible $0.1 \% \mathrm{MUE}$ for tri- and tetravalent lanthanide compounds. Note that the MUE for zero-valent compounds with def2-QZVPPD basis sets is again outside of the target accuracy. The complete results are shown in the Section S4 of the SM.

The MUE of atomic calculations is $8.0 \%$ with def2-SVPD basis sets (see Table II), just at the threshold of the target accuracy, even though we should keep in mind the considerable differences in basis set convergence between $4 \mathrm{f}^{n} 5 \mathrm{~d}^{1}$ and $4 \mathrm{f}^{n+1}$ states, as discussed above. The MUEs of atomic calculations with def2-TZVPPD and def2-QZVPPD basis sets are outside the target accuracy. Therefore, we define the extended def2-TZVPPDD and def2QZVPPDD basis sets specifically for accurate calculations of lanthanide atoms and neutral metal clusters. In these basis sets, the diffuse augmentation is increased to 1p1d1f for the elements $\mathrm{Ce}-\mathrm{Nd}$ and Gd-Tm. 1d1f diffuse set is used in def2-TZVPPDD basis sets for $\mathrm{Pm}-\mathrm{Sm}$ and Er-Tm, while the larger 1p1d1f augmentation is needed in def2-QZVPPDD basis sets for these elements. In addition, 1p augmentation is included in the Eu def2TZVPPDD basis set, and $1 \mathrm{f}$ diffuse set is used in the extended augmentation for Lu. With these additions, the MUE in atomic polarizabilities is $2.3 \%$ with def2-TZVPPDD basis sets. 
The MUE for zero-valent lanthanide compounds is $2.1 \%$ with def2-TZVPPDD basis sets, compared to $8.4 \%$ for def2-TZVPPD. Both these values are within the $2.5 \%$ target accuracy for augmented triple-zeta valence basis sets. The def2-QZVPPDD basis sets produce results very close to the basis set limit: $0.2 \%$ MUE for lanthanide atoms, and $0.4 \%$ for zero-valent lanthanide compounds. The influence of the additional augmentation is quite small in the compounds of lanthanides in positive oxidation states, see Fig. 2. The extended basis sets are thus not necessary in these cases.

The property-optimized basis sets are included in the SM and are available from the Basis Set Exchange online service. ${ }^{76,77}$

TABLE I. Augmentation patterns of property-optimized basis sets for Ce-Lu.

\begin{tabular}{|c|c|c|}
\hline & \multicolumn{2}{|c|}{ Augmentation } \\
\hline & \multicolumn{2}{|c|}{ SVPD TZVPPD QZVPPD } \\
\hline $\mathrm{Ce}-\mathrm{Pr}$ & $1 \mathrm{~d} 1 \mathrm{f}$ & $1 \mathrm{~d} 1 \mathrm{f}$ \\
\hline $\mathrm{Nd}-\mathrm{Sm}, \mathrm{Gd}-\mathrm{Tm}$ & $1 d$ & $1 d$ \\
\hline $\mathrm{Eu}, \mathrm{Yb}$ & - & $1 \mathrm{p}$ \\
\hline \multirow[t]{3}{*}{$\mathrm{Lu}$} & - & - \\
\hline & \multicolumn{2}{|c|}{ Augmentation } \\
\hline & \multicolumn{2}{|c|}{ TZVPPDD QZVPPDD } \\
\hline $\mathrm{Ce}-\mathrm{Nd}, \mathrm{Gd}-\mathrm{Ho}$ & 1p1d1f & $1 \mathrm{p} 1 \mathrm{~d} 1 \mathrm{f}$ \\
\hline $\mathrm{Pm}-\mathrm{Sm}, \mathrm{Er}-\mathrm{Tm}$ & $1 \mathrm{~d} 1 \mathrm{f}$ & $1 \mathrm{p} 1 \mathrm{~d} 1 \mathrm{f}$ \\
\hline $\mathrm{Eu}$ & $1 \mathrm{p}$ & $1 \mathrm{p}$ \\
\hline $\mathrm{Yb}$ & - & $1 \mathrm{p}$ \\
\hline $\mathrm{Lu}$ & $1 \mathrm{f}$ & $1 f$ \\
\hline
\end{tabular}


TABLE II. Statistics of relative errors (in \%) of static isotropic UHF polarizabilities $\alpha_{\text {iso }}$ of $\mathrm{Ce}-\mathrm{Yb}$ atoms in their lowest $4 \mathrm{f}^{n} 5 \mathrm{~d}^{1}$ and $4 \mathrm{f}^{n+1}$ states (except $\operatorname{Tm} 4 \mathrm{f}^{12} 5 \mathrm{~d}^{1}$ ).

\begin{tabular}{|c|c|c|c|c|c|c|c|c|}
\hline & SVP & SVPD & TZVPP & TZVPPD & TZVPPDD & QZVPP & QZVPPD & QZVPPDD \\
\hline Mean & -12.9 & -7.9 & -8.3 & -4.4 & -0.5 & -4.8 & -3.1 & -0.2 \\
\hline Mean uns. & 12.2 & 8.0 & 8.3 & 4.6 & 2.3 & 4.8 & 3.1 & 0.2 \\
\hline Max neg. & -27.7 & -22.5 & -17.6 & -11.1 & -3.9 & -8.7 & -6.9 & -0.9 \\
\hline Max pos. & 0.5 & 0.6 & $\ldots$ & 1.9 & 12.2 & $\ldots$ & 0.0 & 0.1 \\
\hline
\end{tabular}

TABLE III. Statistics of relative errors (in \%) of static isotropic UHF polarizabilities $\alpha_{\text {iso }}$ of the molecular test set (70 molecules).

\begin{tabular}{|c|c|c|c|c|c|c|c|c|}
\hline & SVP & SVPD & TZVPP & TZVPPD & TZVPPDD & QZVPP & QZVPPD & QZVPPDD \\
\hline Mean & -22.6 & -4.1 & -12.4 & -1.8 & -0.7 & -6.1 & -0.8 & -0.2 \\
\hline Mean uns. & 22.6 & 4.4 & 12.4 & 1.9 & 0.9 & 6.1 & 0.9 & 0.3 \\
\hline \multirow[t]{2}{*}{ Max neg. } & -51.5 & -18.8 & -21.5 & -16.0 & -5.4 & -16.6 & -7.7 & -2.5 \\
\hline & DyF & $\mathrm{Gd}_{2}$ & $\mathrm{Gd}_{2}$ & $\mathrm{Gd}_{2}$ & $\mathrm{EuCl}$ & $\mathrm{Gd}_{2}$ & $\mathrm{Gd}_{2}$ & $\mathrm{ErF}$ \\
\hline \multirow[t]{2}{*}{ Max pos. } & 0.8 & 9.2 & 0.1 & 1.4 & 1.5 & $\ldots$ & 1.5 & 0.2 \\
\hline & GdF & $\mathrm{TbO}_{2}$ & $\mathrm{Lu}_{2} \mathrm{~N}$ & DyO & DyO & $\ldots$ & $\mathrm{ErCl}_{2}$ & $\mathrm{TbH}_{3}$ \\
\hline
\end{tabular}




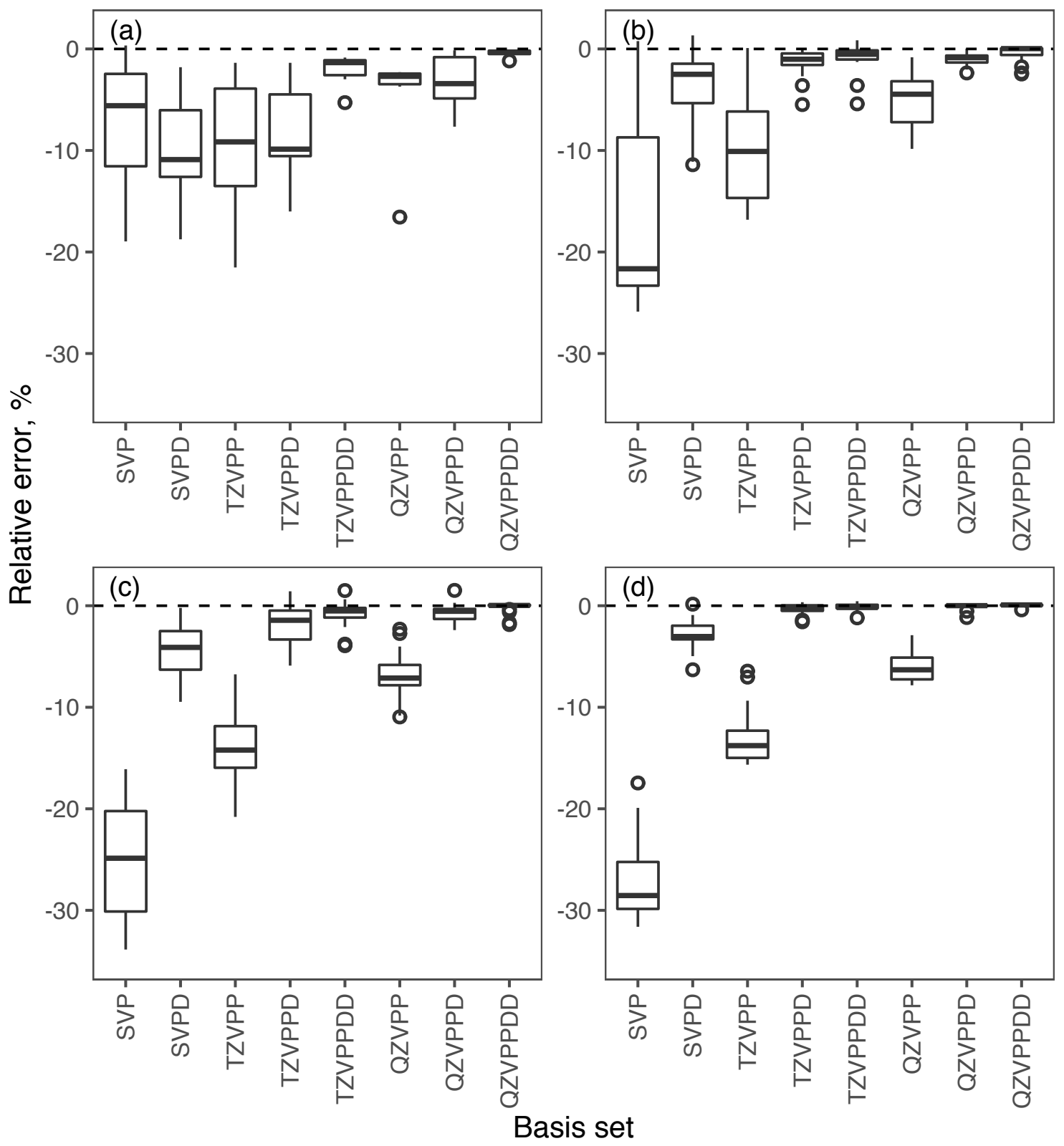

FIG. 2. Summary of relative errors (in \%) of static isotropic UHF polarizabilities $\alpha_{\text {iso }}$ of (a) zerovalent lanthanide compounds (6 molecules), (b) monovalent lanthanide compounds (16 molecules), (c) divalent lanthanide compounds (27 molecules), and (d) tri- and tetravalent lanthanide compounds (21 molecules). The median error within each group is shown by a thick horizontal line, while the box covers the interquartile range between the first and third quartiles. The vertical lines (whiskers) show minimum and maximum values, excluding outliers. Outliers are shown by empty circles. 


\section{APPLICATIONS TO LOW-VALENT LANTHANIDE COMPLEXES}

The chemistry of lanthanide complexes in the +2 oxidation state, which was previously limited to $\mathrm{Eu}, \mathrm{Yb}$, and $\mathrm{Sm}$, has been increasingly extended to other lanthanides. ${ }^{40,78-80}$ One of the most complete series of low-valent lanthanide complexes is $\operatorname{LnCp}_{3}^{\prime-}\left(\mathrm{Cp}^{\prime}=\mathrm{C}_{5} \mathrm{H}_{4} \mathrm{SiMe}_{3}\right)$, which have been characterized for all lanthanides (except for radioactive Pm) ${ }^{38-40,81}$ DFT studies showed that in the non-traditional $\mathrm{LnCp}_{3}^{\prime-}$ complexes $(\mathrm{Ln}=\mathrm{Ce}-\mathrm{Nd}, \mathrm{Gd}-\mathrm{Er}$ ) the highest occupied molecular orbitals (HOMOs) have $\mathrm{d}_{z^{2}}$ character, while the traditional complexes (in the sense of being part of better-known +2 chemistry, $\mathrm{Ln}=\mathrm{Sm}-\mathrm{Eu}, \mathrm{Tm}-\mathrm{Yb}$ ) contain lanthanide +2 ions in their $4 \mathrm{f}^{n+1}$ configurations. The $4 \mathrm{f}^{n+1}$ and $4 \mathrm{f}^{n} 5 \mathrm{~d}^{1}$ configurations are close in energy in the $\mathrm{Ln}=\mathrm{Nd}$ and Dy complexes, however these complexes were assigned $4 \mathrm{f}^{n} 5 \mathrm{~d}^{1}$ configurations on the basis of their x-ray crystal structures and electronic absorption spectra. ${ }^{39}$ The differences in $\mathrm{Ln}-\left(\mathrm{Cp}^{\prime}\right)$ centroid distances between the $\mathrm{LnCp}_{3}^{\prime-}$ complexes and their $\mathrm{LnCp}_{3}^{\prime}$ precursors, which contain +3 lanthanides, was found to be small $(0.02-0.04$ $\AA$ ) when the $\mathrm{Ln}^{+2}$ ions were in $4 \mathrm{f}^{n} 5 \mathrm{~d}^{1}$ configurations and usually larger $(0.05-0.2 \AA)$ for the $\mathrm{Ln}^{+2}$ ions with $4 \mathrm{f}^{n+1}$ configurations. ${ }^{38-40,81}$ Moreover, complexes with $4 \mathrm{f}^{n} 5 \mathrm{~d}^{1}$ configurations show broad absorption bands in the UV/VIS range, in contrast to weaker and sharper electronic transitions typical of $4 \mathrm{f}^{n+1}$ configurations of +2 and +3 lanthanides.

The $\mathrm{LnCp}_{3}^{\prime-}$ complexes provide a robust test set for the property-optimized basis sets due to the competition of the $4 \mathrm{f}^{n+1}$ and $4 \mathrm{f}^{n} 5 \mathrm{~d}^{1}$ configurations within the same series and its experimentally visible effects. We examine the basis set dependence of electronic excitations and UV/VIS absorption spectra of $\mathrm{LnCp}_{3}^{\prime-}$ complexes with $\mathrm{Ln}=\mathrm{Ce}-\mathrm{Nd}, \mathrm{Sm}^{38,39}$ using time-dependent DFT (TDDFT). Of these four complexes, the ones with $\mathrm{Ln}=\mathrm{Ce}-\mathrm{Pr}$ have $4 \mathrm{f}^{n} 5 \mathrm{~d}^{1}$ configurations $(n=1-2)$, the Sm complex has a $4 \mathrm{f}^{n+1}$ configuration $(n=5)$, while the $\mathrm{Nd}$ complex is close to the configuration crossover point, with experimental data supporting the $4 \mathrm{f}^{n} 5 \mathrm{~d}^{1}$ configuration $(n=3) .{ }^{39}$ The optimized ground-state structures of the $\mathrm{Ln}=\mathrm{Pr}-\mathrm{Nd}$, Sm complexes were obtained from Refs. 38 and 39. For $\mathrm{Ln}=\mathrm{Ce}$, the anion geometry was extracted from the $\mathrm{x}$-ray structure ${ }^{39}$ (after removing counterions) and re-optimized with DFT using TPSSh hybrid functional ${ }^{82}$, small-core ECPs ${ }^{32}$ and def2-SVP basis sets. ${ }^{30,31}$ The average distance $\mathrm{Ce}-\left(\mathrm{Cp}^{\prime}\right)$ centroid distance in the optimized structure was $2.56 \AA$, effectively unchanged from the experimental distance. ${ }^{39}$ The RI- $J$ approximation and optimized auxiliary basis sets were used throughout. ${ }^{83,84}$ The effect of solution in THF 
was approximated by the COSMO implicit solvent model ${ }^{85}$ with dielectric constant $\epsilon=$ 7.52. The optimized structure was confirmed to be an energy minimum by force constant calculations. 86,87

TDDFT calculations of electronic excitation spectra ${ }^{88-90}$ were performed at the optimized structures of the $\mathrm{LnCp}_{3}^{\prime-}$ complexes $\left(\mathrm{Ln}=\mathrm{Ce}-\mathrm{Nd}\right.$, Sm) with the PBE0 hybrid functional ${ }^{91}$. We used the unaugmented def2-SVP and def2-TZVP basis sets as well as property-optimized def2-SVPD and def2-TZVPD basis sets. The latter was obtained by adding the diffuse augmentation of the def2-TZVPPD basis set to the smaller def2-TZVP basis sets. The oscillator strengths of electronic transitions were computed in the length gauge. COSMO corrections with the optical index of refraction $n=1.405$ for THF were included in the response calculation. The spectral shapes were simulated from the resulting stick spectrum by empirical Gaussian broadening with $0.2 \mathrm{eV}$ linewidth. The results are presented in Fig. 3. For reference, experimental spectra from Refs. 38 and 39 are shown. The complete results are given in the Section S6 of the SM.

As observed experimentally, the UV/VIS absorption spectra of $\mathrm{LnCp}_{3}^{\prime-}$ complexes ( $\mathrm{Ln}$ $=\mathrm{Ce}-\mathrm{Nd}$ ) containing lanthanide +2 ions in their $4 \mathrm{f}^{n} 5 \mathrm{~d}^{1}$ configurations are dominated by intense transitions from the HOMO orbital, which has $\operatorname{Ln} 5 \mathrm{~d}_{z^{2}}$ character. ${ }^{38-40,81}$ The experimental UV/VIS spectrum of the $\mathrm{CeCp}_{3}^{\prime-}$ complex shows three major absorption bands at $635 \mathrm{~nm}, 540 \mathrm{~nm}$, and $385 \mathrm{~nm}$ with a possible shoulder around $800 \mathrm{~nm}$, see Fig. 3(a). ${ }^{39}$ TDDFT results using def2-SVP basis sets miss the lower-energy features and contain two absorption bands at $759 \mathrm{~nm}$ and around $440 \mathrm{~nm}$, respectively. Additional spectral features appear with def2-SVPD basis sets, with an absorption at $566 \mathrm{~nm}$, which can be tentatively assigned to the experimental band at $540 \mathrm{~nm}$. The main spectral features are qualitatively unchanged with triple-zeta basis sets, which have diffuse augmentation on Ce atom (denoted as TZVP(D) in Fig. 3), however the excitation energies are blue-shifted relative to def2-SVPD results. The lowest-energy band at ca. $760 \mathrm{~nm}$ in the spectrum of the $\mathrm{CeCp}_{3}^{\prime-}$ complex is due to Laporte-allowed Ce $\mathrm{d} \rightarrow \mathrm{f}$ transitions, while the higher-energy transitions consist of combinations of Ce $\mathrm{d} \rightarrow \mathrm{f}$ and metal-ligand charge-transfer (MLCT) transitions into $\mathrm{Cp}^{\prime} \pi^{*}$ orbitals. The spectral shape of the $\mathrm{CeCp}_{3}^{\prime-} \mathrm{UV} / \mathrm{VIS}$ absorption spectrum is well reproduced with augmented double- and triple-zeta basis sets, however, the experimental excitation energies are somewhat overestimated, most likely due to method errors of the PBE0 functional and COSMO implicit solvation. 

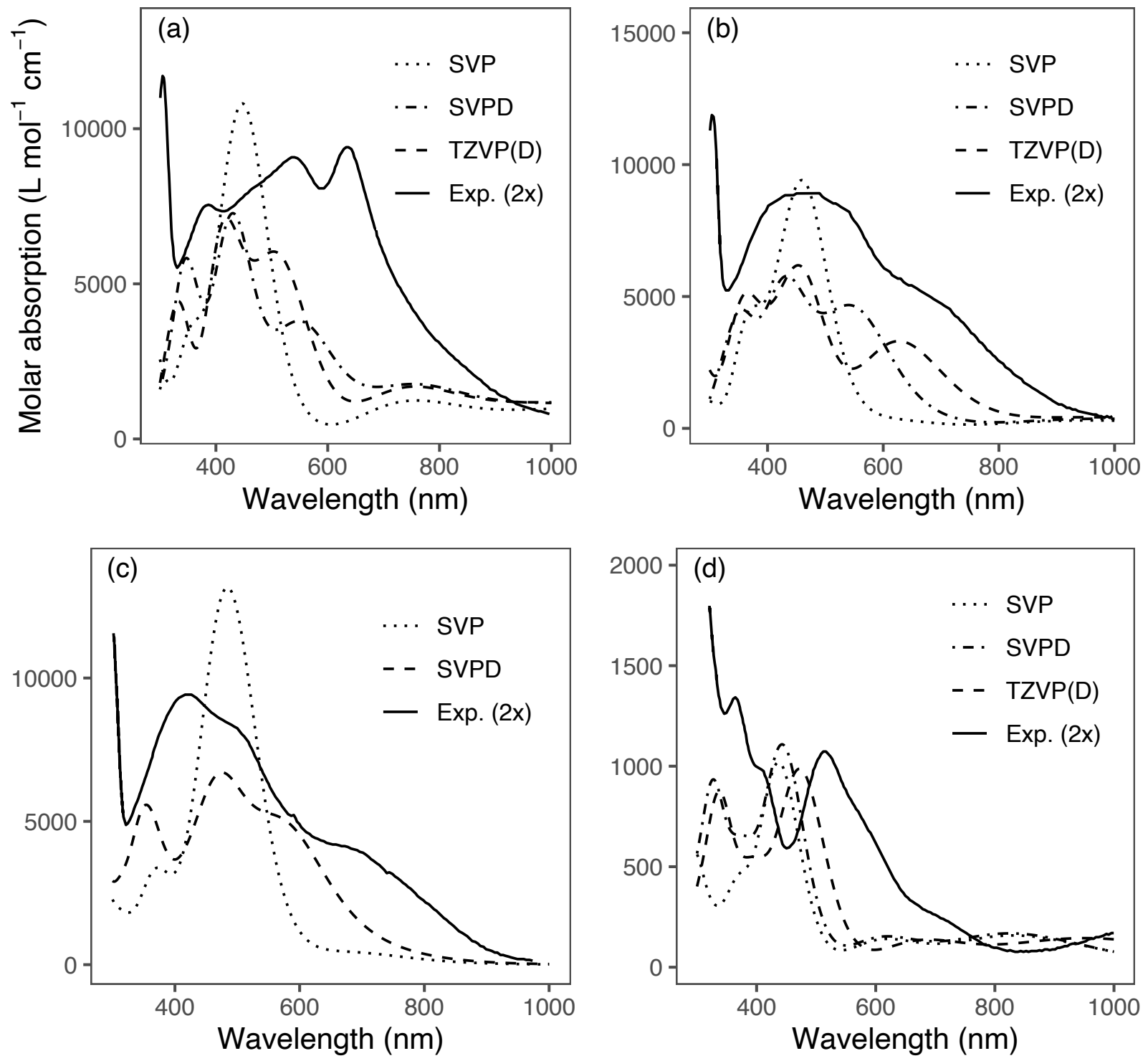

FIG. 3. Experimental and computed UV/VIS absorption spectra of $\mathrm{LnCp}_{3}^{\prime-}$ complexes with $\mathrm{Ln}$ $=(\mathrm{a}) \mathrm{Ce},(\mathrm{b}) \operatorname{Pr},(\mathrm{c}) \mathrm{Nd}$, (d) Sm in THF. The experimental spectra are scaled 2x for clarity. See text for details.

The $\operatorname{PrCp}_{3}^{\prime-}$ complex shows an absorption band at ca. $700 \mathrm{~nm}$ and a broad absorption peak between $410-540 \mathrm{~nm}$ in the experiment, see Fig. 3(b). ${ }^{38}$ As for Ce, the lowest-energy absorption band is absent in the TDDFT results with def2-SVP basis sets and appears only upon augmentation. The TZVP(D) results are in good agreement with the experimental absorption spectra, apart from a blue-shift by ca. $0.15 \mathrm{eV}$. The $700 \mathrm{~nm}$ absorption band is assigned to transitions from Pr d into vacant Pr p orbitals. The broad absorption at higher 
energies arises from transitions from the Pr d orbital into $\operatorname{Pr} \mathrm{f}, \mathrm{Cp}^{\prime} \pi^{*}$ and Rydberg orbitals.

The borderline $\mathrm{NdCp}_{3}^{\prime-}$ complex exists in both $4 \mathrm{f}^{3} 5 \mathrm{~d}^{1}$ and $4 \mathrm{f}^{4}$ configuration, the latter being more stable by merely $7 \mathrm{kcal} / \mathrm{mol}$ at the def2-SVP level. Consistent with Ref. 39, the predicted absorption spectrum of the higher-lying $4 \mathrm{f}^{3} 5 \mathrm{~d}^{1}$ has higher intensity and is in better agreement with the experiment. However, this state becomes unstable with def2-TZVP basis sets, with or without augmentation. The results for the def2-SVP and def2-SVPD basis sets are shown in Fig. 3(c). The experimental absorption spectrum features three bands at 700 $\mathrm{nm}, 510 \mathrm{~nm}$, and $420 \mathrm{~nm}$. As with the neighboring $\operatorname{LnCp}_{3}^{\prime-}$ complexes ( $\left.\mathrm{Ln}=\mathrm{Ce}-\mathrm{Pr}\right)$, the low-energy band is absent with def2-SVP basis sets. The def2-SVPD calculation predicts three main bands in good agreement with experiment. The low-energy absorption consists of $\mathrm{Nd} \mathrm{d} \rightarrow$ p transitions, while the higher-energy bands have MLCT character.

The absorption spectrum of the $\mathrm{SmCp}_{3}^{\prime-}$ complex is much lower in intensity, which is typical of the complexes with the $4 \mathrm{f}^{n+1}$ configuration, as shown in Fig. $3(\mathrm{~d}) .{ }^{39}$ As expected from our small-molecule results, the basis set effects are considerably smaller for this complex due to lack of the highly polarizable 5 d orbital. The two experimental absorption bands at $515 \mathrm{~nm}$ and $410 \mathrm{~nm}$ are well reproduced already at the def2-SVP level. Augmentation does not change the qualitative spectral shape below $400 \mathrm{~nm}$, while the extension to TZVP(D) basis sets results in a small red shift of the lower-energy band. The weak absorptions in the $\mathrm{SmCp}_{3}^{\prime-}$ complex arise from $\mathrm{Sm} \mathrm{f} \rightarrow \mathrm{d}$ transitions with additional MLCT contributions, as expected from absorption data of divalent lanthanides in crystals. ${ }^{92}$

\section{DISCUSSION}

The basis set requirements of response properties of lanthanides and their compounds turn out to be both more element- and oxidation state-dependent than those in main-group elements and transition metals. ${ }^{37}$ However, most of the observed trends can be understood in terms of the competing $4 \mathrm{f}$ and $5 \mathrm{~d}$ occupations. The linear response of an orbital with angular momentum quantum number $l$ to an electric dipole perturbation corresponds to $(l+1)$ and $(l-1)$ quantum numbers (the latter only for $l>0) .{ }^{93}$ The $6 \mathrm{~s}, 5 \mathrm{~d}$, and $4 \mathrm{f}$ subshells are energetically close in lanthanides, however, their spatial extent decreases significantly in the

order $6 \mathrm{~s} \gg 5 \mathrm{~d} \gg 4 \mathrm{f} .{ }^{4,6}$ The $1 \mathrm{p}$ diffuse augmentation obtained by optimizing $4 \mathrm{f}^{n+1}$ reference states thus reflects the orbital response of the 6 s valence orbitals and does not translate into 
improved basis set convergence of lanthanide compounds, in which $6 \mathrm{~s}$ orbitals are typically vacant. On the other hand, diffuse augmentation optimized using $4 \mathrm{f}^{n} 5 \mathrm{~d}^{1}$ reference states is determined by the lanthanide $5 \mathrm{~d}$ orbitals. The largest improvements in atomic polarizabilities are from 1d diffuse augmentation, which corrects the description of the outlying part of the $5 \mathrm{~d}$ orbitals. Further augmentation by $1 \mathrm{f}$ and $1 \mathrm{p}$ diffuse basis functions captures the response of the lanthanide $5 \mathrm{~d}$ and $6 \mathrm{~s}$ orbitals. The occupation and the spatial extent of the $5 \mathrm{~d}$ orbitals are the determining factors for the basis set convergence of polarizabilities in lanthanide compounds. While the trivalent and tetravalent lanthanide compounds require little to no diffuse augmentation on the lanthanide atoms, diffuse basis functions are absolutely crucial for low-valent small molecules and organometallic complexes of lanthanides. The basis set requirements of property calculations in these groups of compounds are well covered by the default property-optimized def2-SVPD, def2-TZVPPD, and def2-QZVPPD basis sets. Therefore, the extended basis sets def2-TZVPPDD and def2-QZVPPDD should only be needed for a relatively narrow class of accurate calculations involving lanthanide atoms or neutral clusters.

Electron correlation and relativistic effects play an important role in polarizabilities of lanthanide atoms. ${ }^{94,95}$ The basis set optimizations presented in this work are based on (unrestricted) $\mathrm{HF}$ with relativistic $\mathrm{ECPs}^{32}$ and thus neglect electron correlation completely while treating relativistic effects at the scalar-relativistic level only. Thierfelder and Schwerdtfeger showed that scalar-relativistic effects at the DK2 level reduce the polarizability of the $\mathrm{Yb}^{1} S$ state from the non-relativistic HF value of $\alpha_{\text {iso }}=230.1$ a. u. to 179.8 a. u. with DK2-HF, ${ }^{94}$ primarily due to the contraction of the $6 \mathrm{~s}^{2}$ subshell. Our HF basis set limit estimate of $\alpha_{\text {iso }}=190.4$ a. u. for $\mathrm{Yb}$ indicates that scalar-relativistic effects are only partially captured by the relativistic ECP. Electron correlation is another possible source of errors in polarizability calculations of lanthanide compounds. The inclusion of electron correlation at the non-relativistic $\operatorname{CCSD}(\mathrm{T})$ level reduces the polarizability of the $\mathrm{Yb}{ }^{1} S$ state by additional 40.9 a.u. ${ }^{94}$ The effect of electron correlation thus seems to be much more significant in the $\mathrm{Yb}$ atom compared to main-group compounds, which showed only a 3.9\% error for static $\mathrm{HF}$ polarizability calculations compared to $\operatorname{CCSD}(\mathrm{T})$ reference results in a recent benchmark of 145 carbon-containing molecules. ${ }^{96}$ On average, our HF basis set limit estimates for $\mathrm{Ce}-\mathrm{Yb}$ (excluding $\mathrm{Lu}$ due to ground-state stability issues) overshoot the best-estimate literature values $^{95}$ by $32 \%$. 
As the example of $\mathrm{LnCp}_{3}^{\prime-}$ complexes $(\mathrm{Ln}=\mathrm{Ce}-\mathrm{Nd}$, Sm) shows, $5 \mathrm{~d}$ occupation is far from rare in low-valent lanthanide compounds. The response of the occupied $\mathrm{d}_{z^{2}}$ orbitals

gives rise to novel optical ${ }^{38-40,66,81}$ and magnetic ${ }^{65,67,97,98}$ properties. Diffuse augmentation on lanthanide atoms is essential for accurate modeling of these properties. The augmented def2-SVPD, def2-TZVPPD, and def2-QZVPPD basis sets are well suited for this task. Interestingly, the UV/VIS absorption spectra of some $\mathrm{LnCp}_{3}^{\prime-}$ complexes feature relatively intense Ln $\mathrm{d} \rightarrow \mathrm{p}$ transitions, as discussed in Section IV and the Section S6 of the SM. In all complexes studied in this work, the experimental absorption spectra were well reproduced without addition of diffuse $\mathrm{p}$ functions, which make only a minor contribution to the response of atomic $4 \mathrm{f}^{n} 5 \mathrm{~d}^{1}$ states, as can be seen from Fig. 1.

Although the property-optimized basis sets were developed by considering atomic polarizabilities at the HF level, they also perform very well for other molecular properties, for example, nonbonding interaction energies, electron affinities, and ground- and exited-state dipole moments, using HF, DFT, and correlated methods. ${ }^{99}$ Due to the balanced construction of the property-optimized basis sets for lanthanides, we expect them to have a similar breadth of applications.

\section{CONCLUSIONS}

We have developed property-optimized basis sets for the lanthanides Ce-Lu for use with small-core ECPs. Contrary to their reputation, these elements show an enormous variety in their response properties as a function of their oxidation state and $5 \mathrm{~d}$ occupation. The default augmented basis sets for lanthanides balance the accuracy across the various bonding situations and provide $\leq 8 \%$ accuracy with def2-SVPD basis sets, $\leq 2.5 \%$ with def2TZVPPD basis sets, and $\leq 1 \%$ for def2-QZVPPD basis sets on the molecular test set, while keeping the size of the diffuse augmentation small. For accurate calculations of lanthanide atoms and neutral clusters, the default augmented basis sets are insufficient. The extended def2-TZVPPDD and def2-QZVPPDD basis sets include additional diffuse augmentations and are recommended for these applications. 


\section{SUPPLEMENTARY MATERIAL}

See the supplementary material for UHF energies and static isotropic polarizabilities and of the molecular test set, Cartesian coordinates of the molecular test set, and electronic excitations in $\mathrm{LnCp}_{3}^{\prime-}$ complexes $\left(\mathrm{Cp}^{\prime}=\mathrm{C}_{5} \mathrm{H}_{4} \mathrm{SiMe}_{3}, \mathrm{Ln}=\mathrm{Ce}-\mathrm{Nd}, \mathrm{Sm}\right)$.

\section{ACKNOWLEDGMENTS}

This work was supported by the National Science Foundation under Grant No. OAC1835909. The computations performed in this work utilized the high-performance computing infrastructure provided by the Research Cyberinfrastructure Center (RCIC) at the University of California, Irvine (UCI), https://rcic.uci.edu. The author thanks Filipp Furche and William J. Evans for helpful discussions.

\section{DATA AVAILABILITY}

The data that support the findings of this study are available within the article and its supplementary material.

\section{REFERENCES}

${ }^{1}$ L. Brewer, "Systematics and the properties of the lanthanides," in Systematics and the Properties of the Lanthanides, edited by S. P. Sinha (Springer, Dordrecht, 1983) pp. 17-69.

${ }^{2}$ J. E. Sansonetti and W. C. Martin, "Handbook of basic atomic spectroscopic data," J. Phys. Chem. Ref. Data 34, 1559-2259 (2005).

${ }^{3}$ K. Balasubramanian, "Relativistic effects and electronic structure of lanthanide and actinide molecules," in Handbook on the Physics and Chemistry of Rare Earths, Vol. 18, edited by K. A. Gschneidner, Jr., L. Eyring, G. R. Choppin, and G. H. Lander (Elsevier, Amsterdam, 1994) Chap. 119, pp. 29-158.

${ }^{4}$ M. Dolg and H. Stoll, "Electronic structure calculations for molecules containing lanthanide atoms," in Handbook on the Physics and Chemistry of Rare Earths, Vol. 22, edited by K. A. Gschneidner, Jr. and L. Eyring (Elsevier, Amsterdam, 1996) Chap. 152, pp. 607-729. 
${ }^{5}$ M. Dolg, ed., Computational Methods in Lanthanide and Actinide Chemistry (Wiley, Chichester, 2018).

${ }^{6}$ K. A. Peterson and K. G. Dyall, "Gaussian basis sets for lanthanide and actinide elements: Strategies for their development and use," in Computational Methods in Lanthanide and Actinide Chemistry, edited by M. Dolg (Wiley, Chichester, 2015) Chap. 8, pp. 195-216.

${ }^{7}$ F. Weigend, "Error-balanced segmented contracted Gaussian basis sets. A concept and its extension to the lanthanides," in Computational Methods in Lanthanide and Actinide Chemistry, edited by M. Dolg (Wiley, Chichester, 2015) Chap. 7, pp. 181-194.

${ }^{8}$ K. A. Peterson and J. G. Hill, "On the development of accurate Gaussian basis sets for f-block elements," Annu. Rep. Comput. Chem. 14, 47-74 (2018).

${ }^{9}$ T. Tsuchiya, M. Abe, T. Nakajima, and K. Hirao, "Accurate relativistic Gaussian basis sets for $\mathrm{H}$ through $\mathrm{Lr}$ determined by atomic self-consistent field calculations with the third-order Douglas-Kroll approximation," J. Chem. Phys. 115, 4463-4472 (2001).

${ }^{10}$ T. Nakajima and K. Hirao, "Accurate relativistic Gaussian basis sets determined by the third-order Douglas-Kroll approximation with a finite-nucleus model," J. Chem. Phys. 116, 8270 (2002).

${ }^{11}$ B. O. Roos, R. Lindh, P.-Å. Malmqvist, V. Veryazov, P.-O. Widmark, and A. C. Borin, "New relativistic atomic natural orbital basis sets for lanthanide atoms with applications to the Ce diatom and $\mathrm{LuF}_{3}$," J. Phys. Chem. A 112, 11431-11435 (2008).

${ }^{12}$ D. A. Pantazis and F. Neese, "All-electron scalar relativistic basis sets for the lanthanides," J. Chem. Theory Comput. 5, 2229-2238 (2009).

${ }^{13}$ D. Aravena, F. Neese, and D. A. Pantazis, "Improved segmented all-electron relativistically contracted basis sets for the lanthanides," J. Chem. Theory Comput. 12, 1148-1156 (2016).

${ }^{14}$ M. Dolg, "Segmented contracted Douglas-Kroll-Hess adapted basis sets for lanthanides," J. Chem. Theory Comput. 7, 3131-3142 (2011).

${ }^{15}$ M. Sekiya, T. Noro, T. Koga, and T. Shimazaki, "Relativistic segmented contraction basis sets with core-valence correlation effects for atoms ${ }_{57} \mathrm{La}$ through ${ }_{71} \mathrm{Lu}$ : Sapporo-DK-nZP sets (n = D, T, Q)," Theor. Chem. Acc. 131, 1247 (2012).

${ }^{16} \mathrm{Q}$. Lu and K. A. Peterson, "Correlation consistent basis sets for lanthanides: The atoms La-Lu," J. Chem. Phys. 145, 054111 (2016).

${ }^{17}$ F. E. Jorge, L. S. C. Martins, and M. L. Franco, "All-electron double zeta basis sets for the lanthanides: Application in atomic and molecular property calculations," Chem. Phys. 
Lett. 643, 84-88 (2016).

${ }^{18}$ A. Z. de Oliveira, I. B. Ferreira, C. T. Campos, F. E. Jorge, and P. A. Fantin, "Segmented all-electron basis sets of triple zeta quality for the lanthanides: Application to structure calculations of lanthanide monoxides," J. Mol. Model. 25, 38 (2019).

${ }^{19}$ I. B. Ferreira, C. T. Campos, and F. E. Jorge, "All-electron basis sets augmented with diffuse functions for $\mathrm{He}, \mathrm{Ca}, \mathrm{Sr}, \mathrm{Ba}$, and lanthanides: Application in calculations of atomic and molecular properties," J. Mol. Model. 26, 95 (2020).

${ }^{20}$ A. S. P. Gomes, K. G. Dyall, and L. Visscher, "Relativistic double-zeta, triple-zeta, and quadruple-zeta basis sets for the lanthanides La-Lu," Theor. Chem. Acc. 127, 369-381 (2010).

${ }^{21}$ J. P. Zobel, P.-O. Widmark, and V. Veryazov, "The ANO-R basis set," J. Chem. Theory Comput. 16, 278-294 (2020).

${ }^{22} \mathrm{P}$. Pollak and F. Weigend, "Segmented contracted error-consistent basis sets of double- and triple- $\zeta$ valence quality for one- and two-component relativistic all-electron calculations," J. Chem. Theory Comput. 13, 3696-3705 (2017).

${ }^{23}$ Y. J. Franzke, L. Spiske, P. Pollak, and F. Weigend, "Segmented contracted error-consistent basis sets of quadruple- $\zeta$ valence quality for one- and two-component relativistic allelectron calculations," J. Chem. Theory Comput. 16, 5658-5674 (2020).

${ }^{24}$ T. R. Cundari and W. J. Stevens, "Effective core potential methods for the lanthanides," J. Chem. Phys. 98, 5555-5565 (1993).

${ }^{25}$ R. B. Ross, S. Gayen, and W. C. Ermler, "Ab initio relativistic effective potentials with spin-orbit operators. V. Ce through Lu," J. Chem. Phys. 100, 8145-8155 (1994).

${ }^{26}$ M. Hülsen, A. Weigand, and M. Dolg, "Quasirelativistic energy-consistent 4f-in-core pseudopotentials for tetravalent lanthanide elements," Theor. Chem. Acc. 122, 23-29 (2009).

${ }^{27}$ M. Hülsen, M. Dolg, P. Link, and U. Ruschewitz, "Improved valence basis sets for divalent lanthanide 4f-in-core pseudopotentials," Theor. Chem. Acc. 129, 367-379 (2011).

${ }^{28} \mathrm{X}$. Cao and M. Dolg, "Valence basis sets for relativistic energy-consistent small-core lanthanide pseudopotentials," J. Chem. Phys. 115, 7348-7355 (2001).

${ }^{29}$ X. Cao and M. Dolg, "Segmented contraction scheme for small-core lanthanide pseudopotential basis sets," J. Mol. Struct. THEOCHEM 581, 139-147 (2002).

${ }^{30}$ R. Gulde, P. Pollak, and F. Weigend, "Error-balanced segmented contracted basis sets of Double- $\zeta$ to Quadruple- $\zeta$ valence quality for the lanthanides," J. Chem. Theory Comput. 
8, 4062-4068 (2012).

${ }^{31} \mathrm{~F}$. Weigend and R. Ahlrichs, "Balanced basis sets of split valence, triple zeta valence and quadruple zeta valence quality for H to Rn: Design and assessment of accuracy," Phys. Chem. Chem. Phys. 7, 3297-3305 (2005).

${ }^{32}$ M. Dolg, H. Stoll, and H. Preuss, "Energy adjusted ab initio pseudopotentials for the rare earth elements," J. Chem. Phys. 90, 1730-1734 (1989).

${ }^{33}$ J. G. Hill, "Gaussian basis sets for molecular applications," Int. J. Quant. Chem. 113, 21-34 (2013).

${ }^{34}$ F. Jensen, "Atomic orbital basis sets," WIREs Comput. Mol. Sci. 3, 273-295 (2013).

${ }^{35}$ B. Nagy and F. Jensen, "Basis sets in quantum chemistry," in Reviews in Computational Chemistry, Vol. 30, edited by A. L. Parrill and K. B. Lipkowitz (Wiley, Hoboken NJ, 2018) Chap. 3, pp. 93-149.

${ }^{36}$ A. A. Buchachenko, G. Chałasiński, and M. M. Szcześniak, "Diffuse basis functions for small-core relativistic pseudopotential basis sets and static dipole polarizabilities of selected lanthanides La, Sm, Eu, Tm and Yb," Struct. Chem. 18, 769-772 (2007).

${ }^{37}$ D. Rappoport and F. Furche, "Property-optimized Gaussian basis sets for molecular response calculations," J. Chem. Phys. 133, 134105 (2010).

${ }^{38}$ M. R. MacDonald, J. E. Bates, J. W. Ziller, F. Furche, and W. J. Evans, "Completing the series of +2 ions for the lanthanide elements: Synthesis of molecular complexes of $\operatorname{Pr}^{2+}$, $\mathrm{Gd}^{2+}, \mathrm{Tb}^{2+}$, and $\mathrm{Lu}^{2+}, "$ J. Am. Chem. Soc. 135, 9857-9868 (2013).

${ }^{39}$ M. E. Fieser, M. R. MacDonald, B. T. Krull, J. E. Bates, J. W. Ziller, F. Furche, and W. J. Evans, "Structural, spectroscopic, and theoretical comparison of traditional vs recently discovered $\mathrm{Ln}^{2+}$ ions in the $[\mathrm{K}(2.2 .2$-cryptand $)]\left[\left(\mathrm{C}_{5} \mathrm{H}_{4} \mathrm{SiMe}_{3}\right)_{3} \mathrm{Ln}\right]$ complexes: The variable nature of $\mathrm{Dy}^{2+}$ and $\mathrm{Nd}^{2+}, "$ J. Am. Chem. Soc. 137, 369-382 (2015).

${ }^{40}$ D. H. Woen and W. J. Evans, "Expanding the +2 oxidation state of the rare-earth metals, uranium, and thorium in molecular complexes," in Handbook on the Physics and Chemistry of Rare Earths, Vol. 50, edited by J.-C. G. B. K. A. Gschneidner, Jr. and V. K. Pecharsky (Elsevier, Amsterdam, 2016) pp. 337-394.

${ }^{41}$ J. O. Hirschfelder, W. Byers Brown, and S. T. Epstein, "Recent developments in perturbation theory," in Advances in Quantum Chemistry, Vol. 1, edited by P.-O. Löwdin (Academic Press, New York, 1964) pp. 255-374. 
${ }^{42}$ E. A. Hylleraas, "Über den Grundterm der Zweielektronenprobleme von $\mathrm{H}^{-}, \mathrm{He}, \mathrm{Li}^{+}, \mathrm{Be}^{++}$ usw." Z. Phys. 65, 209-225 (1930).

${ }^{43}$ R. Jurgens-Lutovsky and J. Almlöf, "Dual basis sets in calculations of electron correlation," Chem. Phys. Lett. 178, 451-454 (1991).

${ }^{44}$ L. Maschio and B. Kirtman, "Coupled perturbation theory approach to dual basis sets for molecules and solids. 1. General theory and application to molecules," J. Chem. Theory Comput. 16, 340-353 (2019).

${ }^{45}$ D. Rappoport, "Basis-set quality and basis-set bias in molecular property calculations," ChemPhysChem 12, 3404-3413 (2011).

${ }^{46}$ F. Furche, B. T. Krull, B. D. Nguyen, and J. Kwon, "Accelerating molecular property calculations with nonorthonormal Krylov space methods," J. Chem. Phys. 144, 174105 (2016).

${ }^{47}$ J. Čížek and J. Paldus, "Stability conditions for the solutions of the Hartree-Fock equations for atomic and molecular systems. application to the Pi-electron model of cyclic polyenes," J. Chem. Phys. 47, 3976-3985 (1967).

${ }^{48}$ D. J. Thouless, "Stability conditions and nuclear rotations in the Hartree-Fock theory," Nucl. Phys. 21, 225-232 (1960).

${ }^{49}$ R. Bauernschmitt and R. Ahlrichs, "Stability analysis for solutions of the closed shell Kohn-Sham equation," J. Chem. Phys. 104, 9047-9052 (1996).

${ }^{50}$ A. D. Becke, "Density-functional exchange-energy approximation with correct asymptotic behavior," Phys. Rev. A 38, 3098-3100 (1988).

${ }^{51}$ J. P. Perdew, "Density-functional approximation for the correlation energy of the inhomogeneous electron gas," Phys. Rev. B 33, 8822-8824 (1986).

${ }^{52}$ F. Weigend, F. Furche, and R. Ahlrichs, "Gaussian basis sets of quadruple zeta valence quality for atoms H-Kr," J. Chem. Phys. 119, 12753-12762 (2003).

${ }^{53}$ A. E. Reed, R. B. Weinstock, and F. Weinhold, "Natural population analysis," J. Chem. Phys. 83, 735-746 (1985).

${ }^{54}$ S. G. Balasubramani, G. P. Chen, S. Coriani, M. Diedenhofen, M. S. Frank, Y. J. Franzke, F. Furche, R. Grotjahn, M. E. Harding, C. Hättig, A. Hellweg, B. Helmich-Paris, C. Holzer, U. Huniar, M. Kaupp, A. M. Khah, S. K. Khani, T. Müller, F. Mack, B. D. Nguyen, S. M. Parker, E. Perlt, D. Rappoport, K. Reiter, S. Roy, M. Rückert, G. Schmitz, M. Sierka, E. Tapavicza, D. P. Tew, C. van Wüllen, V. K. Voora, F. Weigend, A. Wodyński, and 
J. M. Yu, "TURBOMOLE: Modular program suite for ab initio quantum-chemical and condensed-matter simulations," J. Chem. Phys. 152, 184107 (2020).

${ }^{55}$ TURBOMOLE V7.5 2020, a development of University of Karlsruhe and Forschungszentrum Karlsruhe GmbH, 1989-2007, TURBOMOLE GmbH, since 2007; available from https://www.turbomole.org.

${ }^{56}$ M. Dolg, H. Stoll, and H. Preuss, "Homonuclear diatomic lanthanoid compounds: a pseudopotential configuration interaction and correlation energy density functional study," J. Mol. Struct. THEOCHEM 277, 239-249 (1992).

${ }^{57}$ X. Cao and M. Dolg, "Electronic structure of lanthanide dimers," Mol. Phys. 101, 1967-1976 (2003).

${ }^{58}$ J. R. Lombardi and B. Davis, "Periodic properties of force constants of small transitionmetal and lanthanide clusters," Chem. Rev. 102, 2431-2460 (2002).

${ }^{59}$ J. O. Kafader, J. E. Topolski, and C. C. Jarrold, "Molecular and electronic structures of cerium and cerium suboxide clusters," J. Chem. Phys. 145, 154306 (2016).

${ }^{60} \mathrm{~B}$. Roos and P. Pyykkö, "Bonding trends in molecular compounds of lanthanides: The double-bonded carbene cations $\mathrm{LnCH}_{2}^{+}$( $\left.\mathrm{Ln}=\mathrm{Sc}, \mathrm{Y}, \mathrm{La}-\mathrm{Lu}\right)$," Chem. Eur. J. 16, 270-275 (2010).

${ }^{61} \mathrm{~W}$. Xu, X. Jin, M. Chen, P. Pyykkö, M. Zhou, and J. Li, "Rare-earth monocarbonyls MCO: comprehensive infrared observations and a transparent theoretical interpretation for $\mathrm{M}=\mathrm{Sc}$; Y; La-Lu," Chem. Sci. 3, 1548-1554 (2012).

${ }^{62}$ F. G. N. Cloke, "Zero oxidation state compounds of scandium, yttrium, and the lanthanides," Chem. Soc. Rev. 22, 17-24 (1993).

${ }^{63}$ G. B. Deacon and Q. Shen, "Complexes of lanthanoids with neutral $\pi$ donor ligands," J. Organomet. Chem. 511, 1-17 (1996).

${ }^{64}$ G. Hong, F. Schautz, and M. Dolg, "Ab initio study of metal-ring bonding in the bis $\left(\eta^{6}\right.$ benzene)lanthanide and -actinide complexes $\mathrm{M}\left(\mathrm{C}_{6} \mathrm{H}_{6}\right)_{2}(\mathrm{M}=\mathrm{La}, \mathrm{Ce}, \mathrm{Nd}, \mathrm{Gd}, \mathrm{Tb}, \mathrm{Lu}, \mathrm{Th}$, U)," J. Am. Chem. Soc. 121, 1502-1512 (1999).

${ }^{65}$ K. R. Meihaus, M. E. Fieser, J. F. Corbey, W. J. Evans, and J. R. Long, "Record high single-ion magnetic moments through $4 \mathrm{f}^{n} 5 \mathrm{~d}^{1}$ electron configurations in the divalent lanthanide complexes $\left[\left(\mathrm{C}_{5} \mathrm{H}_{4} \mathrm{SiMe}_{3}\right)_{3} \mathrm{Ln}\right]^{-}$," J. Am. Chem. Soc. 137, 9855-9860 (2015).

${ }^{66}$ A. J. Ryan, L. E. Darago, S. G. Balasubramani, G. P. Chen, J. W. Ziller, F. Furche, J. R. Long, and W. J. Evans, "Synthesis, structure, and magnetism of tris(amide) 
$\left[\operatorname{Ln}\left\{\mathrm{N}\left(\mathrm{SiMe}_{3}\right)_{2}\right\}_{3}\right]^{1-}$ complexes of the non-traditional +2 lanthanide ions," Chem. Eur. J. 24, 7702-7709 (2018).

${ }^{67}$ C. A. Gould, K. R. McClain, J. M. Yu, T. J. Groshens, F. Furche, B. G. Harvey, and J. R. Long, "Synthesis and magnetism of neutral, linear metallocene complexes of Terbium(II) and Dysprosium(II)," J. Am. Chem. Soc. 141, 12967-12973 (2019).

${ }^{68}$ X. Shen, L. Fang, X. Chen, and J. R. Lombardi, "Absorption, excitation, and resonance raman spectra of $\mathrm{Ce}_{2}, \mathrm{Pr}_{2}$, and $\mathrm{Nd}_{2}$," J. Chem. Phys. 113, 2233-2237 (2000).

${ }^{69}$ M. Dolg, H. Stoll, and H. Preuss, "Pseudopotential study on rare earth dihalides and trihalides," J. Mol. Struct. THEOCHEM 235, 67-79 (1991).

${ }^{70}$ T. R. Cundari, S. O. Sommerer, L. A. Strohecker, and L. Tippett, "Effective core potential studies of lanthanide complexes," J. Chem. Phys. 103, 7058-7063 (1995).

${ }^{71}$ T. Tsuchiya, T. Taketsugu, H. Nakano, and K. Hirao, "Theoretical study of electronic and geometric structures of a series of lanthanide trihalides $\operatorname{LnX}_{3}(\mathrm{Ln}=\mathrm{La}-\mathrm{Lu} ; \mathrm{X}=\mathrm{Cl}, \mathrm{F})$, ” . Mol. Struct. THEOCHEM 461, 203-222 (1999).

${ }^{72}$ W. Xu, W.-X. Ji, Y.-X. Qiu, W. H. E. Schwarz, and S.-G. Wang, "On structure and bonding of lanthanoid trifluorides $\mathrm{LnF}_{3}$ (Ln = La to Lu)," Phys. Chem. Chem. Phys. 15, 7839-7847 (2013).

${ }^{73}$ Y. Wang and M. Dolg, "Pseudopotential study of the ground and excited states of $\mathrm{Yb}_{2}$," Theor. Chem. Acc. 100, 124-133 (1998).

${ }^{74}$ A. A. Buchachenko, G. Chałasiński, and M. M. Szcześniak, "Europium dimer: van der Waals molecule with extremely weak antiferromagnetic spin coupling," J. Chem. Phys. 131, $241102(2009)$.

${ }^{75}$ P. Schwerdtfeger, O. R. Smits, and P. Pyykkö, "The periodic table and the physics that drives it," Nat. Rev. Chem. 4, 359-380 (2020).

${ }^{76}$ B. P. Pritchard, D. Altarawy, B. Didier, T. D. Gibson, and T. L. Windus, "New basis set exchange: An open, up-to-date resource for the molecular sciences community," J. Chem. Inf. Model. 59, 4814-4820 (2019).

${ }^{77}$ Basis Set Exchange, developed as a collaboration between the Molecular Sciences Software Institute (MolSSI) and the Pacific Northwest National Lab/Environmental Molecular Sciences Laboratory (PNNL/EMSL), https://www. basissetexchange org.

${ }^{78}$ M. N. Bochkarev, "Molecular compounds of "new" divalent lanthanides," Coord. Chem. Rev. 248, 835-851 (2004). 
${ }^{79}$ F. Nief, "Molecular chemistry of the rare-earth elements in uncommon low-valent states," in Handbook on the Physics and Chemistry of Rare Earths, Vol. 40, edited by K. A. Gschneidner, Jr., J.-C. G. Bünzli, and V. K. Pecharsky (Elsevier, Amsterdam, 2010) Chap. 246, pp. 241-300.

${ }^{80}$ R. Collins, J. P. Durrant, M. He, and R. A. Layfield, "Electronic structure and magnetic properties of rare-earth organometallic sandwich compounds," in Handbook on the Physics and Chemistry of Rare Earths, Vol. 55, edited by J.-C. G. Bünzli and V. K. Pecharsky (Elsevier, Amsterdam, 2019) Chap. 307, pp. 89-121.

${ }^{81}$ M. R. MacDonald, J. E. Bates, M. E. Fieser, J. W. Ziller, F. Furche, and W. J. Evans, "Expanding rare-earth oxidation state chemistry to molecular complexes of Holmium(II) and Erbium(II)," J. Am. Chem. Soc. 134, 8420-8423 (2012).

${ }^{82}$ V. N. Staroverov, G. E. Scuseria, J. Tao, and J. P. Perdew, "Comparative assessment of a new nonempirical density functional: Molecules and hydrogen-bonded complexes," J. Chem. Phys. 119, 12129-12137 (2003).

${ }^{83}$ K. Eichkorn, O. Treutler, H. Öhm, M. Häser, and R. Ahlrichs, "Auxiliary basis sets to approximate Coulomb potentials (Chem. Phys. Letters 240 (1995) 283-290)," Chem. Phys. Lett. 242, 652-660 (1995).

${ }^{84}$ F. Weigend, "Accurate Coulomb-fitting basis sets for H to Rn," Phys. Chem. Chem. Phys. 8, 1057-1065 (2006).

${ }^{85}$ A. Klamt and G. Schüurmann, "COSMO: a new approach to dielectric screening in solvents with explicit expressions for the screening energy and its gradient," J. Chem. Soc. Perkin Trans. 2 , 799-805 (1993).

${ }^{86}$ P. Deglmann, F. Furche, and R. Ahlrichs, "An efficient implementation of second analytical derivatives for density functional methods," Chem. Phys. Lett. 362, 511-518 (2002).

${ }^{87}$ P. Deglmann, K. May, F. Furche, and R. Ahlrichs, "Nuclear second analytical derivative calculations using auxiliary basis set expansions," Chem. Phys. Lett. 384, 103-107 (2004).

${ }^{88}$ R. Bauernschmitt and R. Ahlrichs, "Treatment of electronic excitations within the adiabatic approximation of time dependent density functional theory," Chem. Phys. Lett. 256, 454-464 (1996).

${ }^{89}$ R. Bauernschmitt, M. Häser, O. Treutler, and R. Ahlrichs, "Calculation of excitation energies within time-dependent density functional theory using auxiliary basis set expansions," Chem. Phys. Lett. 264, 573-578 (1997). 
${ }^{90}$ F. Furche and D. Rappoport, "III. Density functional methods for excited states: Equilibrium structure and electronic spectra," in Computational Photochemistry, Vol. 16, edited by M. Olivucci (Elsevier, Amsterdam, 2005) pp. 93-128.

${ }^{91}$ J. P. Perdew, M. Ernzerhof, and K. Burke, "Rationale for mixing exact exchange with density functional approximations," J. Chem. Phys. 105, 9982-9985 (1996).

${ }^{92}$ G. H. Diecke, H. M. Crosswhite, and H. Crosswhite, Spectra and Energy Levels of Rare Earth Ions in Crystals (Wiley-Interscience, New York, 1968).

${ }^{93}$ A. J. Sadlej, "Medium-size polarized basis sets for high-level correlated calculations of molecular electric properties," Coll. Czech. Chem. Commun. 53, 1995-2016 (1988).

${ }^{94}$ C. Thierfelder and P. Schwerdtfeger, "Effect of relativity and electron correlation in static dipole polarizabilities of ytterbium and nobelium," Phys. Rev. A 79, 032512 (2009).

${ }^{95}$ P. Schwerdtfeger and J. K. Nagle, "2018 Table of static dipole polarizabilities of the neutral elements in the periodic table," Mol. Phys. 117, 1200-1225 (2018).

${ }^{96}$ T. Wu, Y. N. Kalugina, and A. J. Thakkar, "Choosing a density functional for static molecular polarizabilities," Chem. Phys. Lett. 635, 257-261 (2015).

${ }^{97}$ V. Dubrovin, A. A. Popov, and S. Avdoshenko, "Magnetism in ln molecular systems with 4f/valence-shell interplay (FV-magnetism)," Chem. Commun. 55, 13963-13966 (2019).

${ }^{98}$ V. E. Fleischauer, G. Ganguly, D. H. Woen, N. J. Wolford, W. J. Evans, J. Autschbach, and M. L. Neidig, "Insight into the electronic structure of formal lanthanide(II) complexes using magnetic circular dichroism spectroscopy," Organometallics 38, 3124-3131 (2019).

${ }^{99}$ A. Hellweg and D. Rappoport, "Development of new auxiliary basis functions of the Karlsruhe segmented contracted basis sets including diffuse basis functions (def2-SVPD, def2TZVPPD, and def2-QVPPD) for RI-MP2 and RI-CC calculations," Phys. Chem. Chem. Phys. 17, 1010-1017 (2014). 